\title{
Joint Channel, Carrier-Frequency-Offset and Noise-Variance Estimation for OFDM Systems Based on Expectation Maximization
}

\author{
Jiankang Zhang ${ }^{\dagger *}$, Xiaomin $\mathrm{Mu}^{\dagger}$, Lajos Hanzo* \\ $\dagger$ School of Information Engineering, Zhengzhou University, Zhengzhou, China, \\ * School of ECS, University of Southampton, SO17 1BJ, United Kingdom. \\ Tel: +44-23-8059 3125, Fax: +44-23-8059 4508 \\ Email: jz09v@ecs.soton.ac.uk, iexmmu@zzu.edu.cn, lh@ecs.soton.ac.uk \\ http://www-mobile.ecs.soton.ac.uk
}

\begin{abstract}
In this paper, a joint channel, carrier-frequencyoffset (CFO) and noise-variance estimation scheme is proposed for OFDM systems which is based on Expectation and Maximization (EM) algorithm. The channel parameters are estimated using training sequences incorporated at the beginning of each transmission frame. Based on the assumption that the amplitude and CFO of different paths are independent, the received multipath components may be decomposed into $L$ independent data sets of the $L$ resolvable propagation paths. Hence the associated multi-dimensional minimization problem may be decomposed into separate single-dimensional minimization processes, the maximum likelihood and yet, remains capable of approaching performance at a signifucantly reduced complexity.

Index Terms-OFDM, channel estimation, Expectation and Maximization, time-variant channel.
\end{abstract}

\section{INTRODUCTION}

Orthogonal frequency division multiplexing (OFDM) has received considerable research-attention [1] and has been adopted by numerous standards bodies for both wireline and wireless communications. For example, Digital Subscriber Lines (DSL), the European Digital Audio and Video Broadcast (DAB/DVB) system, Wireless Area Networks (IEEE 802.11a), the IEEE 802.16a standard and Japan's Mobile Multimedia Acess Communication (MMAC) system all employ OFDM.

Accurate channel estimation is necessary for OFDM to coherently demodulate the received data, hence diverse algorithms have been designed, which employed different optimization criteria and imposed varying levels of implementation complexity [1]. As the affordable hardware capacity is increasing, it becomes more feasible to implement iterative receivers allowing for substantial improvements of the physical layer functions [2]. The iterative expectation-maximization (EM) algorithm has been shown to strike an attractive trade-off between the performance attained and the complexity imposed $[3,4,8]$. More specifically, EM estimation was applied to the problem of sequence estimation in fading channels [3]. The authors of [4] proposed three EM-based frequency domain algorithms to estimate either the channel's impulse response (CIR) or the channel's frequency response (CFR) by making use of a modest number of pilot tones. By characterizing

Acknowledgments: The financial support of the China Scholarship Council (CSC) and the EU under the auspices of the OPTIMIX project as well as of the EPSRC, UK is gratefully acknowledged. the channel estimation problem using a state-space model relying on unknown model parameters, Cai et al. [5] applied the EM algorithm to obtain the maximum likelihood (ML) estimates of these unknown parameters. They required no $a$ priori knowledge of the channel's fading statistics. In order to improve attainable transmission efficiency, Zhang et al. [6] proposed a robust EM-based joint channel and carrier frequency offset (CFO) estimation technique based on the ML criterion. The authors of $[9,10]$ applied the EM algorithm to optimize the maximum a posteriori (MAP) criterion. Unbiased EM and unbiased conditional EM (UCEM) algorithms were investigated in [11] for MIMO-OFDM systems.

It is important to emphasize that EM based channel estimation methods typically carry out channel estimation in the frequency domain after the Fast Fourier Transform (FFT) based demodulator. By contrast, the novel contribution of this paper is that we design a joint CIR, CFO and noisevariance estimation technique based on the EM algorithm which processes the received signal before FFT based demodulation in the time domain. The proposed joint CIR, $\mathrm{CFO}$ and noise-variance estimation algorithm requires no pilot information and no a priori knowledge of the channel's fading statistics. And yet it is capable of iteratively generating the ML estimate of the CIR, CFO and noise-variance. This is achieved without requiring the computation of gradients, nor is it necessary to adaptively adjust any step-size parameters.

The rest of the paper is organized as follows. In Section II, the OFDM system model is described. Section III presents the proposed EM channel estimation algorithm. Analysis and discussion are presented in Section IV. In Section V, the performance of the proposed EM estimation algorithm is evaluated by simulation in terms of its normalized meansquared error (NMSE) and bit error ratio (BER). Finally, our summary and concluding remarks are provided in Section VI.

\section{System Model}

A cyclic prefix (CP) based OFDM transceiver scheme is displayed in Fig. 1. We assume that the $\mathrm{CP}$ is sufficiently long to eliminate intersymbol interference (ISI) between consecutive OFDM sysmbols. The number of tones or subcarriers in the system is $N_{c}$ and the length of the CP is $N_{c p}$ samples. 


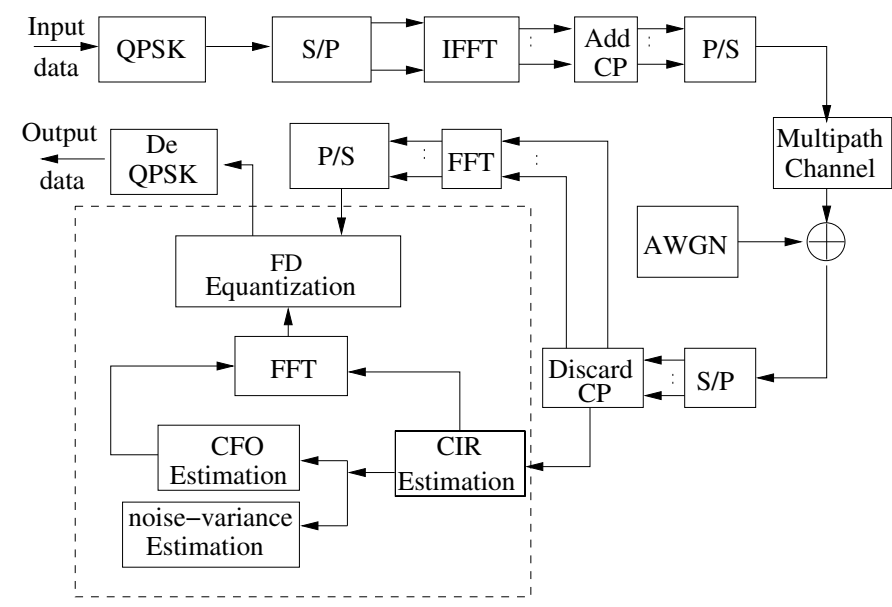

Fig. 1. Baseband OFDM System Model

Under these assumptions we describe the system with the aid of a discrete-time model. The serial input sequence is quaternary phase-shift keying (QPSK) modulated and the QPSK symbols are mapped to the $N_{c}$ subcarriers of the OFDM modulator. The frequency-domain (FD) subcarriersymbols are then transformed to the time-domain (TD) by the unitary Inverse Fast Fourier Transform (IFFT). The basedband TD model of the $n$-th sample of the $m$-th OFDM symbol can be presented as

$$
x_{m}(n)=\operatorname{IFFT}\left\{X_{m}(k)\right\}=\frac{1}{N_{c}} \sum_{k=0}^{N_{c}-1} X_{m}(k) e^{j 2 \pi k n T_{s} / T},
$$

where $T_{s}$ is the OFDM sampling interval and $T=N_{c} T_{s}$ is the time duration of an OFDM symbol without the CP. After concatenating the $\mathrm{CP}$ of length $N_{c p}$ samples, the serially converted data is transmitted through a multipath fading channel and contaminated by the receiver's additive white Gaussian noise (AWGN). In the presence of the CFO $\epsilon_{l}$, which is normalized to the intercarrier spacing, the received symbol $y_{m}(n)$ can then be represented as

$$
y_{m}(n)=\sum_{l=0}^{L-1} h_{m, l} e^{j \frac{2 \pi}{N_{c}} \epsilon_{l} n} x_{m}(n-l)+z_{m}(n) .
$$

To simplify the expressions, we drop the OFDM symbol index $m$ in Equation (2), yielding

$$
y(n)=\sum_{l=0}^{L-1} h_{l} e^{j \frac{2 \pi}{N_{c}} \epsilon_{l} n} x(n-l)+z(n) .
$$

\section{Proposed EM Channel Estimation Scheme}

The EM algorithm constitutes an iterative technique of finding the ML estimates of parameters that is particularly attractive when direct access to the data necessary to make an estimate is unavailable, or when some of the data are missing. The EM algorithm can be broken down into two primary steps, namely that of determining the expectation of the data followed by a maximization step. The expectation is obtained with respect to the underlying unknown variables, using the current estimate of the parameters, which is conditioned upon the observations. The maximization step then provides a new estimate of the parameters. These two steps are activated alternatively, until convergence is attained.

Again, the proposed channel estimation is carried out before the FFT based demodulation, as seen in Fig. 1. The estimated value of CIR, CFO and noise-variance is successively calculated by the three estimator blocks of Fig. 1 and then these parameters are forwarded to the equantization module. Derivation of the proposed EM based channel estimation algorithm is based on the discrete-time received signal model of (3).

The CIR $h_{l}, l=0,1, \cdots, L-1$, CFO $\epsilon_{l}, l=0,1, \cdots, L-1$ and the noise-variance $\sigma^{2}$ have to be estimated, hence we define the three-component parameter set $\boldsymbol{\Psi}=\left\{h_{l}, \epsilon_{l}, \sigma^{2} \mid l=\right.$ $0,1, \cdots, L-1\}$, which has to be estimated. Classic ML channel estimation would require a multi-dimensional minimization of the objective function (OF) formulated as:

$$
J(\Psi)=\sum_{n=0}^{N_{c}-1}\left(y(n)-\sum_{l=0}^{L-1} h_{l} e^{j \frac{2 \pi}{N_{c}} \epsilon_{l} n} x(n-l)\right),
$$

over the entire search-space of the legitimate CIR, CFO and noise-variance range, which may become excessive for a high number of multipath components $L$. We assume that the $L$ taps of $h_{l}$ are independent complex-valued Gaussian, i. e. Rayleigh faided variables. Hence the received signal $y(n)$ may be replaced by the sum of independently faded multipath components $S_{l}(n)$

$$
\begin{aligned}
y(n)= & \sum_{l=0}^{L-1} S_{l}(n), \\
S_{l}(n)= & h_{l} e^{j \frac{2 \pi}{N_{c}} \epsilon_{l} n} x(n-l)+z_{l}(n), \\
& l=0,1, \cdots, L-1,
\end{aligned}
$$

where $z_{l}(n)$ is the AWGN with a variance of $\sigma_{l}^{2}$ and we have $\sum_{l=0}^{L-1} \sigma_{l}^{2}=\sigma^{2}$. Then the problem of estimating the $L$ taps of $h_{l}$ may be decoupled into $L$ independent CIR-tap estimation processes. Hence it may be argued that due to the independence of the $L$ fading taps $h_{l}$ the probability density function (PDF) of $h_{l}$ may be decomposed into the $L$ individual PDFs of the $L$ multipath components, $S_{L}$. Consequently, the ML estimation of the $L$ taps of $h_{l}$ may be decomposed into $L$ individual path-estimation processes formulated as:

$J\left(h_{l}\right)=\sum_{n=0}^{N_{c}-1}\left[S_{l}(n)-h_{l} e^{j \frac{2 \pi}{N_{c}} \epsilon_{l} n} x(n-l)\right]^{2}, l=0,1, \cdots, L-1$.

More explicitly, the original $L$-dimensional minimization problem of Equation (4) has been decomposed into $L$ separate single-dimensional minimization steps, which is in general a more tractable problem. Following the terminology of EM algorithms, the decomposed multipath component set $\mathbf{S}=$ $S_{0}(n), S_{1}(n), \cdots, S_{L-1}(n)$ is referred to as the complete data. 
Originally, our goal was to find the ML estimate of $\Psi$ in Equation (4) by maximizing $p_{\mathbf{y}}(\mathbf{y} ; \mathbf{\Psi})$. However it is too complex to carry out this maximization directly, hence we maximize $\ln p_{\mathbf{S}}(\mathbf{S} ; \boldsymbol{\Psi})$ instead. Because it is more convenient to estimate its $\log$-likelihood function $\ln p_{\mathbf{y}}(\mathbf{y} ; \mathbf{\Psi})$, as this allows us to convert the associated products to additions. Since $\mathbf{S}$ is unavailable, we use the conditional expectation of its loglikelihood function given $\mathbf{y}$, which is formulated as:

$$
E_{\mathbf{S} \mid \mathbf{y}}\left\{\ln p_{\mathbf{S}}(\mathbf{S} ; \mathbf{\Psi})\right\}=\sum_{l=0}^{L-1} \ln p_{\mathbf{S}}\left(S_{l} ; \mathbf{\Psi}\right) p\left(S_{l} \mid \mathbf{y} ; \mathbf{\Psi}\right) .
$$

Clearly, we have to know $\boldsymbol{\Psi}$ in order to determine $p\left(S_{l} \mid \mathbf{y} ; \boldsymbol{\Psi}\right)$ in Equation (8), but $\boldsymbol{\Psi}$ is unknown. Hence, in order to proceed, we adopt its expected value, i. e. the expected loglikelihood function as its current estimate. Letting $\hat{\mathbf{h}}^{(k)}$, $\hat{\epsilon}^{(k)}$ and $\left(\hat{\sigma}^{2}\right)^{(k)}$ denote the $k$ th estimate of the ML estimate of $\mathbf{h}, \epsilon$ and $\sigma^{2}$, where we have $\mathbf{h}=\left[h_{0}, h_{1}, \cdots, h_{L-1}\right], \epsilon=$ $\left[\epsilon_{0}, \epsilon_{1}, \cdots, \epsilon_{L-1}\right]$, the following iterative two-stage algorithm may be contrived:

Expectation calculation Step (E-Step): Determine the average log-likelihood function of the complete data as follows:

$$
U\left(\boldsymbol{\Psi}, \hat{\mathbf{\Psi}}^{(k)}\right)=E\left\{\ln p_{\mathbf{S}}(\mathbf{S} ; \Psi) \mid \mathbf{y} ; \hat{\mathbf{\Psi}}^{(k)}\right\} ;
$$

Maximization Step (M-Step): Maximize the average loglihood function of the complete data over all possible values of $\mathbf{h}$, which is formulated as:

$$
\hat{\mathbf{\Psi}}^{(k+1)}=\arg \max _{\boldsymbol{\Psi}} U\left(\boldsymbol{\Psi}, \hat{\mathbf{\Psi}}^{(k)}\right) .
$$

The detailed derivation of the above-mentioned two-stage channel estimation algorithm based on EM is outlined below.

By exploiting the independent data set assumption of (6), it may be readily shown that we formulate the log-likelihood function of the complete data as follows:

$$
\begin{aligned}
& \ln p_{\mathbf{S}}(\mathbf{S} ; \boldsymbol{\Psi}) \\
= & \sum_{l=0}^{L-1} \ln p\left(S_{l} ; h_{l}\right) \\
= & C-\sum_{l=0}^{L-1} \frac{1}{2 \sigma_{l}^{2}} \sum_{n=0}^{N_{c}-1}\left\|S_{l}(n)-h_{l} e^{j \frac{2 \pi}{N_{c}} \epsilon_{l} n} x(n-l)\right\|^{2} \\
= & g(S)+\sum_{l=0}^{L-1} \frac{1}{2 \sigma_{l}^{2}} \sum_{n=0}^{N_{c}-1}\left[-2 \mathcal{R}\left\{S_{l}^{*}(n) h_{l} e^{j \frac{2 \pi}{N_{c}} \epsilon_{l} n}\right.\right. \\
& \left.x(n-l)\}+\left\|h_{l}\right\|^{2}\|x(n-l)\|^{2}\right],
\end{aligned}
$$

where $C$ is a constant. Following the FFT-based demodulation of the QPSK signal in the schematic of Fig. 1, we have $\sum_{n=0}^{N_{c}-1}\|x(n-l)\|^{2}=N_{c}$. Since we wish to maximize $U\left(\Psi, \hat{\boldsymbol{\Psi}}^{(k)}\right)$ with respect to $\boldsymbol{\Psi}$ in Equation (11), we can omit the expected value of the constant $C$ and $g(S)$, because they do not depend on $\boldsymbol{\Psi}$. Hence we can expand the E-Step (9) as

$$
\begin{aligned}
U\left(\Psi, \hat{\Psi}^{(k)}\right)= & \sum_{l=0}^{L-1} \frac{1}{2 \sigma_{l}^{2}} \sum_{n=0}^{N_{c}-1}\left[-2 \mathcal{R}\left\{\hat{S}_{l}^{(k) *}(n) h_{l} e^{j \frac{2 \pi}{N_{c}} \epsilon_{l} n}\right.\right. \\
& \left.x(n-l)\}+\left\|h_{l}\right\|^{2}\|x(n-l)\|^{2}\right]
\end{aligned}
$$

where we have

$$
\hat{S}_{l}^{(k)}(n)=E\left\{S_{l}(n) \mid y(n) ; \hat{\mathbf{\Psi}}^{(k)}\right\} .
$$

Since $S_{l}(n)$ only depends on the received signal $y(n)$ and they are jointly Gaussian distributed, according to the standard result for the conditional expectations of jointly Gaussian random vectors it may be readily shown that we have:

$$
\hat{S}_{l}^{(k)}(n)=S_{l}^{(k)}(n)+\frac{\hat{\sigma}_{l}^{2}}{\hat{\sigma}^{2}}\left(y(n)-\sum_{l=0}^{L-1} S_{l}^{(k)}(n)\right),
$$

where

$$
S_{l}^{(k)}(n)=\hat{h}_{l}^{(k)} e^{j \frac{2 \pi}{N_{c}} \hat{\epsilon}_{l}^{(k)} n} x(n-l) .
$$

After obtaining $\hat{S}_{l}^{(k)}(n)$ of Equation (14) for all samples of the $n=0,1, \cdots, N_{c}-1$, the $M$-step of Equation 10 aims to calculate the new estimates for the channel taps $\hat{\mathbf{h}}^{(k+1)}$ of the $(k+1)$ st iteration, which may be expressed as

$$
\begin{aligned}
& \hat{\mathbf{h}}^{(k+1)}=\arg \min _{\mathbf{h}} \sum_{l=0}^{L-1} \frac{1}{2 \sigma_{l}^{2}} \sum_{n=0}^{N_{c}-1}\left[-2 \mathcal{R}\left\{\hat{S}_{l}^{(k) *}(n) \hat{h}_{l}^{(k)}\right.\right. \\
& \left.\left.e^{j \frac{2 \pi}{N_{c}} \hat{\epsilon}_{l}^{(k)} n} x(n-l)\right\}+\left\|\hat{h}_{l}^{(k)}\right\|^{2}\|x(n-l)\|^{2}\right],
\end{aligned}
$$

where $\mathcal{R}\{\cdot\}$ denotes the real part of $\{\cdot\}$.

Again, since estimating all the $L$ CIR taps jointly by the EM process imposes a potentially excessive complexity, the $L$-dimensional minimization problem of Equation (16) may be decomposed into $L$ separate single-dimensional minimization steps as

$$
\begin{aligned}
& \hat{h}_{l}^{(k+1)}=\arg \min _{h_{l}} \sum_{n=0}^{N_{c}-1}\left[-2 \mathcal{R}\left\{\hat{S}_{l}^{(k) *}(n) \hat{h}_{l}^{(k)} e^{j \frac{2 \pi}{N_{c}} \hat{\epsilon}_{l}^{(k)} n}\right.\right. \\
& \left.x(n-l)\}+\left\|\hat{h}_{l}^{(k)}\right\|^{2}\|x(n-l)\|^{2}\right], l=0,1, \cdots, L-1 .
\end{aligned}
$$

which yields:

$$
\hat{h}_{l}^{(k+1)}=\frac{1}{N_{c}}\left(\sum_{n=0}^{N_{c}-1} \hat{S}_{l}^{(k) *}(n) e^{j \frac{2 \pi}{N_{c}} \hat{\epsilon}_{l}^{(k)} n} x(n-l)\right)^{*} .
$$

When the CIR has been estimated in the next step, the noisevariance can also be estimated by setting the derivative of $U\left(\Psi, \hat{\Psi}^{(k)}\right)$ with respect to $\sigma_{l}^{2}$ to zero, which yields:

$$
\begin{array}{r}
\left(\hat{\sigma}_{l}^{2}\right)^{(k+1)}=\frac{\sum_{n=0}^{N_{c}-1}\left[-2 \mathcal{R}\left\{\hat{S}_{l}^{(k) *}(n) \hat{h}_{l}^{(k+1)} e^{j \frac{2 \pi}{N_{c}} \hat{\epsilon}_{l}^{(k)} n}\right.\right.}{\sum_{n=0}^{N_{c}-1}\left[-2 \mathcal{R}\left\{\frac{1}{\left(\hat{\sigma}^{2}\right)^{(k)}}\left(y(n)-\sum_{l=0}^{L-1} S_{l}^{(k)}(n)\right)\right.\right.} \\
\cdots \frac{\left.x(n-l)\}+\left\|\hat{h}_{l}^{(k+1)}\right\|^{2}\|x(n-l)\|^{2}\right]}{\left.\left.\hat{h}_{l}^{(k+1)} e^{j \frac{2 \pi}{N_{c}} \hat{\epsilon}_{l}^{(k)} n} x(n-l)\right\}\right]},
\end{array}
$$


where we have $\left(\hat{\sigma}^{2}\right)^{(k)}=\sum_{l=0}^{L-1}\left(\hat{\sigma}_{l}^{2}\right)^{(k)}$.

Similarly, we may arrive at the ML estimate of the CFO $\epsilon_{l}$ as follows:

$$
\hat{\epsilon}_{l}^{(k+1)}=\hat{\epsilon}_{l}^{(k)}-\frac{\sum_{n=0}^{N_{c}-1} 2 \mathcal{R}\left\{\hat{S}_{l}^{(k) *}(n)\left(j \frac{2 \pi}{N_{c}} n\right) e^{j \frac{2 \pi}{N_{c}} \hat{\epsilon}_{l}^{(k)} n} x(n-l)\right\}}{\sum_{n=0}^{N_{c}-1} 2 \mathcal{R}\left\{\hat{S}_{l}^{(k) *}(n)\left(j \frac{2 \pi}{N_{c}} n\right)^{2} e^{j \frac{2 \pi}{N_{c}} \hat{\epsilon}_{l}^{(k)} n} x(n-l)\right\}} .
$$

Obsevere in $U\left(\boldsymbol{\Psi}, \hat{\boldsymbol{\Psi}}^{(k)}\right)$ that the term $e^{j \frac{2 \pi}{N_{c}} \epsilon_{l} n}$ is non-linear, which causes problems in terms of the EM updates between iteration $k$ and $(k+1)$, when computing the CFO estimate $\hat{\epsilon}_{l}^{(k+1)}$. Hence we simplify the expression of $U\left(\boldsymbol{\Psi}, \hat{\mathbf{\Psi}}^{(k)}\right)$ in Equation (12) using the Taylor series expansion of $e^{j \frac{2 \pi}{N_{c}} \epsilon_{l} n}$ around $\hat{\epsilon}_{l}^{(k)}$ and retain its first three terms according to:

$$
\begin{aligned}
e^{j \frac{2 \pi}{N_{c}} \epsilon_{l} n} \approx & e^{j \frac{2 \pi}{N_{c}} \hat{\epsilon}_{l}^{(k)} n}+\left(\epsilon_{l}-\hat{\epsilon}_{l}^{(k)}\right)\left(j \frac{2 \pi}{N_{c}} n\right) e^{j \frac{2 \pi}{N_{c}} \hat{\epsilon}_{l}^{(k)} n} \\
& +\frac{1}{2}\left(\epsilon_{l}-\hat{\epsilon}_{l}^{(k)}\right)^{2}\left(j \frac{2 \pi}{N_{c}} n\right)^{2} e^{j \frac{2 \pi}{N_{c}} \hat{\epsilon}_{l}^{(k)} n}
\end{aligned}
$$

\section{ANALYSIS AND DiscUSSION}

In this section, we evaluate the performance of the proposed scheme and compare it to other EM based CIR estimation methods operating in the FD. Furthermore, we consider the achievable inter-carier interference (ICI) reduction and the complexity of the proposed EM based CIR, CFO and noisevariance estimation technique.

In order to estimate the CIR in the FD, we demodulate the received TD samples $y(n)$ formulated in Equation (2) by taking the FFT, yielding:

$$
\begin{aligned}
Y(n)= & F F T\{y(k)\}=\sum_{k=0}^{N_{c}-1} y(k) e^{-\frac{j 2 \pi k n}{N_{c}}} \\
= & G(n, n) X(n)+\underbrace{\sum_{k=0, k \neq n}^{N_{c}-1} G(n, k) X(k)}_{I C I} \\
& +Z(n), n=0,1, \cdots, N_{c}-1,
\end{aligned}
$$

where we have

$$
\begin{aligned}
Z(n) & =F F T\{z(k)\}=\sum_{k=0}^{N_{c}-1} z(k) e^{\frac{-j 2 \pi k n}{N_{c}}} \\
G(n, k) & =\frac{1}{N_{c}} \sum_{m=0}^{N_{c}-1} \sum_{l=0}^{L-1} h_{l} e^{\frac{j 2 \pi \epsilon_{l} k}{N_{c}}} e^{-\frac{j 2 \pi m(n-k)}{N_{c}}} e^{-\frac{j 2 \pi k l}{N_{c}(24)}}
\end{aligned}
$$

The classic FD channel estimation methods process the received FD signals $\left\{Y(n), n=0,1, \cdots, N_{c}-1\right\}$, which has

\begin{tabular}{|c|c|c|c|}
\hline Algorithm & Products & Additions & $\begin{array}{l}\text { Martix inver- } \\
\text { sion }\end{array}$ \\
\hline $\begin{array}{l}\text { Alg. in } \\
{[3]}\end{array}$ & $\begin{array}{l}2 N_{c}^{3}+N_{c}^{2}+ \\
9 N_{c}\end{array}$ & $2 N_{c}^{3}+3 N_{c}^{2}$ & $\left(N_{c} \times N_{c}\right)_{1}$ \\
\hline $\begin{array}{l}\text { Alg. in } \\
{[5]}\end{array}$ & $\begin{array}{l}\left(15 L^{3}+\right. \\
\left.12 L^{2}+3 L\right) N_{c}\end{array}$ & $\begin{array}{l}\left(16 L^{3}+\right. \\
\left.8 L^{2}+6 L\right) N_{c}\end{array}$ & $(L \times L)_{3}$ \\
\hline $\begin{array}{l}\text { Alg. } 1 \text { in } \\
{[4]}\end{array}$ & $\begin{array}{l}\left(19 N_{s p}\right. \\
1) N_{c}^{2}\end{array}$ & $4 N_{c}^{2}$ & $\left(N_{s p} \times N_{s p}\right)_{N_{c}^{2}}$ \\
\hline $\begin{array}{l}\text { Alg. } 3 \text { in } \\
{[4]}\end{array}$ & $5 N_{c} L+4 L$ & $2 N_{c} L$ & None \\
\hline Proposed & $4 N_{c} L$ & $2 N_{c} L$ & None \\
\hline
\end{tabular}
been contaminated by the ICI term and by the sum of the noise samples accumulated after the FFT operation. By contrast, the proposed EM based CIR estimation technique is not effected by the ICI.

The complexity of the various algorithms considered is summarized find in Table 1,
Table 1 Complexity Comparison

where $N_{s p}$ represents the number of constellation points in the signal-space, i. e. we have $N_{s p}=4$ for QPSK, and $(N \times N)_{m}$ represents carrying out the inversion of a $N \times N$ martix $m$ times.

Compared to the EM-based channel estimation methods of $[4,6,10,11]$, the proposed scheme has the following beneficial features: (1) CIR estimation is carried out in the TD before the FFT based demodulation which allows us to mitigate the effects ICI imposed by the movement of the receiver at the output of the FFT-based demodulator of Fig. 1 as detailed in [1]; (2) The proposed CIR estimation scheme requires less multiplications and additions and avoids the matrix inversion, which significantly reduce the complexity of the estimator; (3) The accuracy of the estimates will not be affected by the CIR length $L$, while in conventional channel estimation schemes the length $L$ of the CIR has a grave effect on the accuracy of the estimation [12]; (4) The classic FD channel estimation has to rely on the knowledge of the number of CIR taps, which determines the required FD pilot-spacing, i. e. the pilot overhead. By contrast, the proposed TD channel estimation scheme only requires a rough estimate of the number of CIR taps $L=\max \left\{l \mid\left\|\hat{h}_{l}\right\|^{2} \geq\right.$ Threshold $\}$.

\section{Simulation Results}

In this section, the performance of the proposed EM based TD CIR, CFO and noise-variance estimator is studied with the aid of simulations. We consider the IEEE 802. 11a WLAN using $N_{c}=64$ subcarriers and a single sample per subcarrier. The length of the $\mathrm{CP}$ is assumed to be $N_{c p}=16$ samples and QPSK is used. The negative exponentially decaying Rayleigh fading CIR has $L=7$ taps, with path delays of $0,1, \cdots, L-1$ samples and a delay profile specified by $E\left\{\alpha_{l}^{2}\right\}=\exp (-l / 10)$. The amplitude $\alpha_{l}$ of each path is independent from those of the others. Hence the CIR used in on simulations is given by

$$
h(n)=\frac{1}{K} \sum_{l=0}^{6} e^{-k / 2} \alpha_{l} \delta(n-l),
$$

where $K=\sqrt{\sum_{l=0}^{6} e^{(-l)}}$ is a normalization factor.

Fig. 2 shows the normalized mean squared error (NMSE) performance versus the SNR for the different channel esti- 


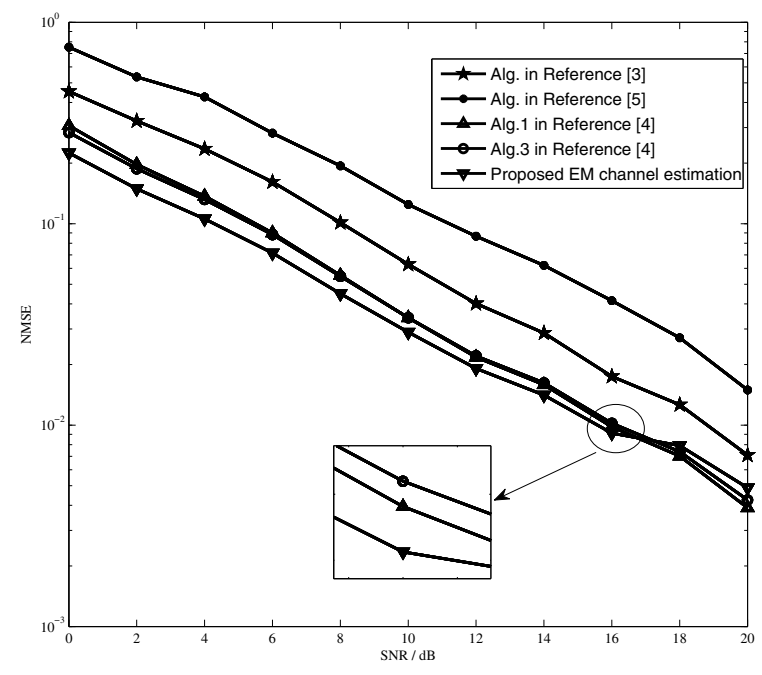

Fig. 2. NMSE performance comparison for various channel estimation algorithms.

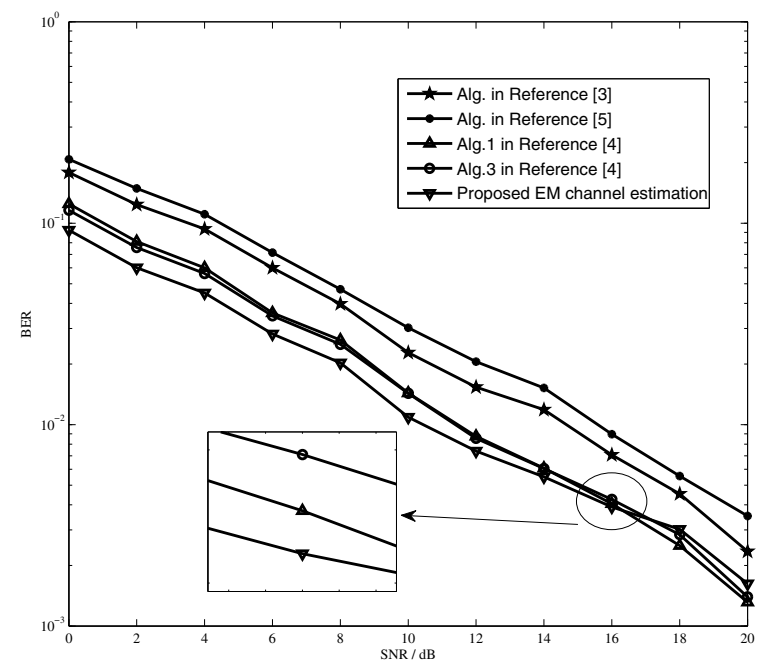

Fig. 3. BER performance comparison for various channel estimation algorithms.

mation schemes considered. We can see that the NMSE performance of the proposed EM based CIR estimation scheme outperforms the Alg. 1 and the Alg. 3 of [4] by $2 d B$ for $0 d B \leq S N R \leq 6 d B$, and it becomes about $1 d B$ better than those of [4] for $6 d B \leq S N R \leq 14 d B$. Compared to the EM based CIR estimation schemes of [3] and [5], the NMSE performance of the proposed scheme exhibits a significant SNR improvement of about $4 d B$. However, the proposed reduced-complexity TD CIR estimation scheme performed worse for $16 d B \leq S N R$ than the FD Alg. 1 and Alg. 3 of [4].

The achievable BER performance is shown in Fig. 3, indicating that the proposed EM based CIR estimation scheme outperforms the existing EM channel estimation schemes for
$S N R \leq 12 d B$, by about $3 d B, 1 d B$ and $4 d B$, respectively, when compared to the algorithms proposed in [3], [4] and [5]. Unfortunately, the reduced-complexity TD scheme performs modestly for $16 d B \leq S N R$, when compared to the highercomplexity FD algorithms of [4], although it retains its advantages compared to the algorithms of [3] and [5].

\section{CONCLUSION}

In this paper we presented a joint EM-based CIR estimation scheme operating in time domain. The channel estimation scheme advocated is capable of interatively estimating the $\mathrm{CIR}, \mathrm{CFO}$ and noise-variance. In contrast to existing EMbased channel estimation methods, the proposed technique operates in the TD, i. e. before FFT-based demodulation, hence it is capable of reducing the ICI.

\section{REFERENCES}

[1] L. Hanzo, M. Munster, B. J. Choi, and T. Keller, OFDM and MC-CDMA for Broadband Multi-User Communications, WLANs and Broadcasting. Piscataway, NJ: IEEE Press, 2003.

[2] Y. Liu, L. Brunel and J.J. Boutros, EM Channel Estimation for Coded OFDM Transmissions over Frequency-Selective Channel, IEEE 10th International Symposium on Spread Spectrum Techniques and Applications, 2008. ISSSTA '08., pp. 544-549.

[3] C. N. Georghiades and C. H. Jae, Sequence Estimation in the Presence of Random Parameters via the EM Algorithm, IEEE Transactions on Communications, vol. 45, pp. 300-308, 1997.

[4] X. Q. Ma, H. Kobayashi and S. C. Schwartz, EM-based Channel Estimation Algorithm for OFDM, Eurasip Journal on Applied Signal processing, vol. 2004, pp. 1460-1477, 2004.

[5] J. Cai, X. Shen and J. W. Mark, EM Channel Estimation Algorithm for OFDM Wireless Communication Systems, 14th IEEE Proceedings of Personal, Indoor and Mobile Radio Communications Confersece, 2003. PIMRC 2003., pp. 804-808.

[6] R. P. Zhang, T. Y. Zhang and Z. W. Gong, EM-based Joint Channel Estimation and Data Detection Algorithm, Journal of Electronics \& Information Technology, vol. 28, pp. 2130-3134, 2006. (in Chinese)

[7] A. Waku, M. Fujii, M. Itami and I. K., A Study on Channel Estimation for OFDM Systems using EM Algorithm Based on Multi-Path Doppler Channel Model, Multi-Carrier Spread Spectrum 2007 Proceedings from the 6th International Workshop on Multi-Carrier Spread Spectrum, pp. 337-346.

[8] R. H. Mo, Y. H. Chew, T. T. Tjhung and C. C. Ko, An EMbased Semiblind Joint Channel and Frequency Offset Estimator for OFDM Systems over Frequency-Selective fading Channels, IEEE Transactions on Vehicular Technology, vol. 57, pp. 32753282, 2008.

[9] H. Dogan, H. A. Cirpan and E. Panayirci, Iterative Channel Estimation and Decoding of Turbo Coded SFBC-OFDM Systems, IEEE Transactions on Wireless Communications, vol. 6, pp. 3090-3101, 2007.

[10] X. Zhang and J. Wei, MAP Receiver with Enhanced EM Channel Estimation for MIMO-OFDM Systems, IEEE Wireless Communications and Networking Conference, 2007. WCNC 2007., pp. 932-936.

[11] X. Wautelet, C. Herzet, A. Dejonghe, J. Louveaux, L. Vandendorpe, Comparison of EM-Based Algorithms for MIMO Channel Estimation, Communications, IEEE Transactions on, vol. 55, pp. 216-226, 2007.

[12] J. Zhang, E. Chen, X. Mu and S. Yang, Tracking Effective Length of Channel Impulse Response for ML Estimator in OFDM Systems, 4th International Conference on Wireless Communications, Networking and Mobile Computing, 2008. WiCOM '08., pp. 1-4. 